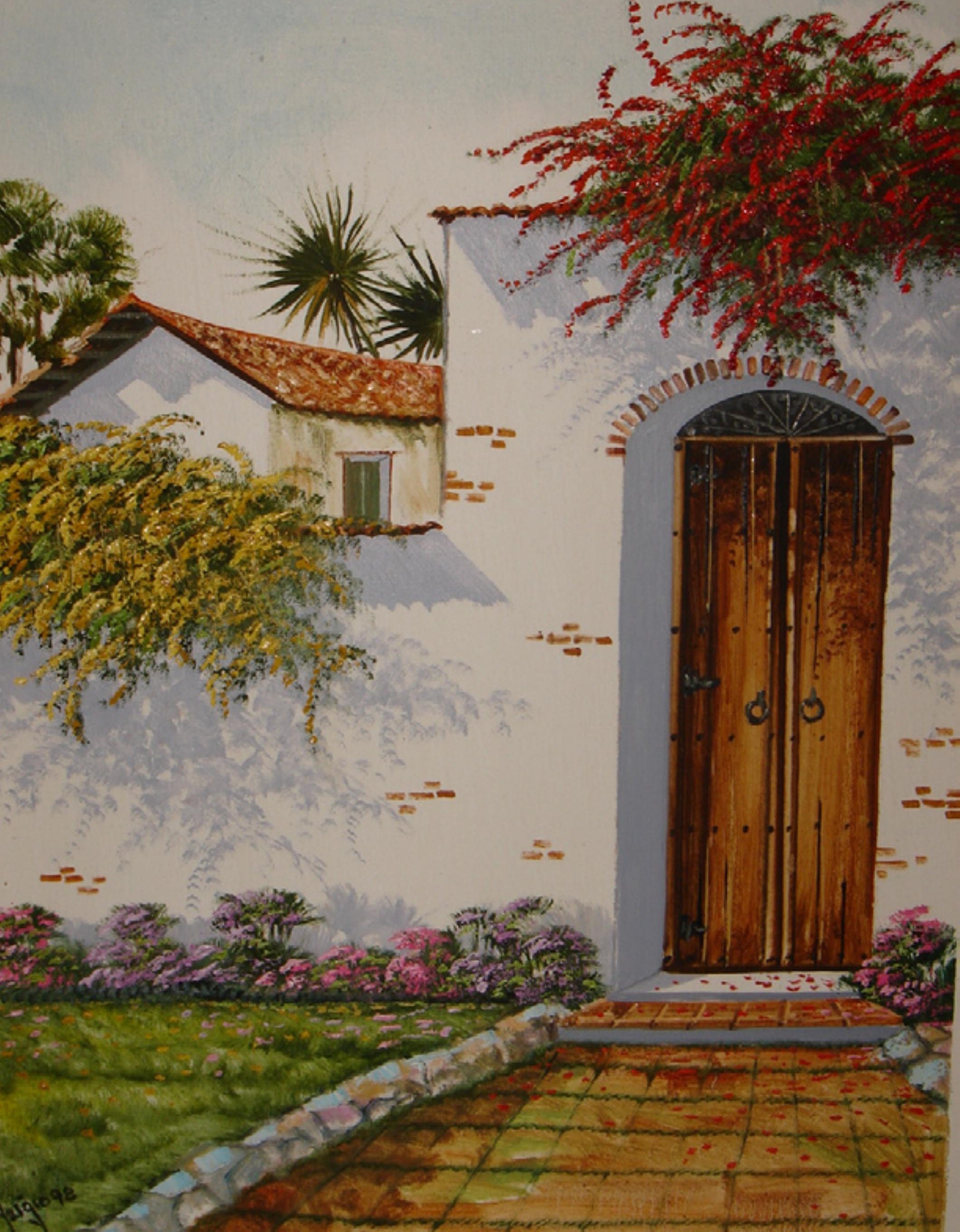




\title{
5 la industria maquiladora de exportación y el territorio en Honduras (el caso de Choloma y Villanueva)
}

\author{
RAFAEl ANTONIO DELGAdO ELVIR
}

\section{CARACTERIZACIÓN DE LA INDUSTRIA MAQUILADORA DE EX- PORTACIÓN EN LOS MUNICIPIOS DE CHOLOMA Y VILLANUEVA}

La industria maquiladora de exportación (IME) surgió y se desarrolló en los municipios de Choloma, Villanueva, San Pedro Sula, La Lima y Puerto Cortés. La cercanía al puerto marítimo, el fácil acceso a éste por medio de buenas vías de comunicación, la existencia de un centro urbano desarrollado como San Pedro Sula y la oferta abundante de mano de obra con poca formación vocacional, facilitó que se desarrollara en el corredor comprendido entre Puerto Cortés y Villanueva un conglomerado de Zonas Libres (ZOLI), Zonas Industriales de Procesamiento (ZIP) y de empresas bajo el Régimen de Importación Temporal (RIT). ${ }^{1}$ Paralelamente, aunque no con el mismo dinamismo, surgieron otras regiones del país atractivas para la inversión en la maquila. Así surgieron maquilas en La Ceiba, El Progreso, Comayagua, Tegucigalpa y recientemente en Quimistán (Santa Bárbara).

A continuación se detallan algunas características básicas que definen la dimensión socioeconómica de la IME establecida en los municipios de Choloma y Villanueva:

a) Origen del capital: En los municipios de Choloma y Villanueva prevalecen las maquiladoras de capital norteamericano, coreano y hondureño. Sin embargo, en Villanueva se muestra una alta concentración de empresas norteamericanas como Sara Lee, Fruit of the Loom, Skips, Garan, Rusell Corporation. ${ }^{2}$ En Choloma por su parte se muestra una presencia destacada de maquiladoras hondureñas, ligadas a los grupos ELCATEX e IHNDELVA y una fuerte presencia de maquiladoras norteamericanas y coreanas (ver Tabla 1 ).

b) Ubicación de las empresas: Se han establecido en los parques industriales (ZOLIs y ZIPs) construidos especialmente para albergar este tipo de industria con una infraestructura de acuerdo a las necesidades de la IME. Los parques industriales, en su mayoría de capital hondureño, además del alquiler de los edificios ofrecen servicios adicionales como ser seguridad, disposición de basura, servicios de agua potable y electricidad, sucursales de bancos, prescreening de empleados entre otros. Estos parques se ubican muy cerca de las cabeceras municipales, a la orilla de la principal vía de comunicación que conecta el país de norte a sur y en especial hacia Puerto Cortés.

1 La Ley de Zonas Libres (1976, 1989, 1994, 1998) la Ley de Zonas Industriales de Procesamiento (1987, ) y el Régimen de Importación Temporal (1984) son los tres regímenes especiales que se han creado para el desarrollo de la Industria Maquila de Exportación (IME). Para ver una breve descripción y alcance de estas leyes y sus reformas, consultar FIDE: Agenda para la Competitividad: La Industria Textil y de la Confección en Honduras. Condiciones Competitivias del Valle de Sula, Marzo 2003.

2 Ver Asociación Hondureña de Maquiladores: Directorio 2003-2004. c) Régimen legal: Por estar establecidas en ZOLI y ZIPs se rigen bajo la Ley de Zonas Libres y la Ley de Zonas Industriales de Procesamiento y sus reformas. Sin embargo, dada las reformas a la Ley de Zonas Libres de 1998, que extiende los beneficios de esta ley a todo el país y ante el vencimiento de los beneficios arancelarios a las empresas dueñas y operadoras de los parques contempladas en la Ley ZIP, los parques ZIP han procedido a acogerse a la Ley ZOLI. No se cuenta con información de empresas que funcionen bajo el Régimen de Importación Temporal (RIT) en estos municipios.

d) Tamaño de las empresas: Serie de datos exactos sobre la producción mensual son muy escasos. Por lo tanto se recurre al empleo como criterio para medir el tamaño. Son empresas intensivas en el uso de mano de obra cuyos tamaños varían considerablemente. Para el año 2003 el tamaño promedio de las empresas en Villanueva (576 operarios por maquiladora) se ubica sobre las empresas de Choloma (446 operarios por maquiladora), influenciado este factor por la presencia en Villanueva de grandes empresas norteamericanas cuyos tamaños oscilan entre 800 a 900 empleados.

e) Tipo de producción: Siguiendo la tendencia observada en otros municipios maquiladores, la IME se ha concentrado en la producción de prendas de vestir y en especial en la producción de camisetas, pantalones cortos, ropa interior y deportiva para niños y adultos.

Tabla 1: Origen, número y origen de las Empresas de la IME en los municipios de Choloma y Villanueva (2003)

\begin{tabular}{lcc|lll} 
& \multicolumn{3}{c|}{ Choloma } & \multicolumn{3}{c}{ Villanueva } \\
\hline Origen & $\begin{array}{c}\text { Empre- } \\
\text { sas }\end{array}$ & Empleo & Origen & $\begin{array}{c}\text { Empre- } \\
\text { sas }\end{array}$ & Empleo \\
Estados & 20 & 12.753 & $\begin{array}{l}\text { Estados } \\
\text { Unidos }\end{array}$ & 31 & 23.675 \\
Unidos & 18 & 10.151 & Taiwan & 2 & 2.278 \\
Honduras & 13 & 5.286 & Hong Kong & 2 & 892 \\
Corea & 2 & 1.289 & Honduras & 2 & 809 \\
$\begin{array}{l}\text { Canada } \\
\text { Honduras/ }\end{array}$ & 1 & 578 & Singapore & 1 & 280 \\
Hong Kong & & 396 & Honduras/ & 1 & 175 \\
Hong Kong & 2 & 42 & USA & & \\
Honduras/ & 1 & & & & \\
China & & $\mathbf{3 0 . 9 9}$ & Total & $\mathbf{3 9}$ & $\mathbf{2 8 . 6 0 9}$ \\
Total & $\mathbf{5 8}$ & &
\end{tabular}

Fuente: Asociación Hondureña de Maquiladores: Directorio 2003-2004. 
f) Empleo: Los municipios de Choloma y Villanueva concentran actualmente alrededor del $57 \%$ del total del empleo de la maquila. Las cifras de empleo en Choloma alcanzaron en el año 1998 un total de 43,808 operarios. Mientras Villanueva, muy por debajo, empleaba 24,810 operarios. Sin embargo, para 2003 el panorama se muestra diferente con 30,990 operarios y 28,609 operarios en Choloma y Villanueva respectivamente (ver Tabla 1 y Tabla 4).

g) Género: Son empresas que emplean predominantemente mano de obra joven femenina, para la realización de operaciones de costura, planchado, inspección y empaque de ropa. Según las últimas cifras de la Asociación Hondureña de Maquiladores la mano de obra femenina constituye alrededor del $60 \%$ del empleo total.

h) Planificación de la producción: La producción y por lo tanto el empleo depende y se desarrolla sobre la base de los requerimientos del mercado norteamericano y en especial del cliente de la IME. La producción en general se planifica sobre la base de contratos o pedidos del cliente en EUA quien envía para el maquilado todos los insumos de la prenda. En el caso de maquilas ligadas a una empresa manufacturera extranjera, la producción se desarrolla en función de los pedidos que realiza la casa matriz. En estas plantas no se desarrollan procesos de investigación, desarrollo ni diseño de productos.

i) Organización flexible de la producción: Los operarios son organizados en grupos, módulos, líneas de producción o células. Cada uno de estos módulos posee las máquinas y los operarios necesarios para realizar todas las operaciones para elaborar la prenda desde el principio hasta el fin. De acuerdo al tipo de prenda y su estilo se cambian el tamaño así como la integración de los módulos.

j) Estándares técnicos y de calidad: Los módulos de trabajo son monitoreados y calificados sobre la base de estrictos criterios de tiempo y calidad. La producción está minuciosamente regulada por los requerimientos de tiempo por tipo de operación (elaboración de ruedo, pegado de cuello, pegado de manga, pegado de etiqueta etc.) En este sentido, los operarios son entrenados para que en un período relativamente corto ( $2-3$ semanas) eleven la intensidad del trabajo (operaciones por unidad de tiempo) al nivel deseado. El control de calidad se lleva a cabo a través de inspecciones concurrentes al producto en proceso y al producto terminado. Se realiza también la auditoría a posteriori por auditores internos de las empresas y por los auditores de los clientes.

k) Remuneración: Los operarios son remunerados semanalmente sobre la base del rendimiento individual y del módulo. Los operarios que no alcanzan las metas de cantidad y calidad y los que se encuentran en período de aprendizaje se les remunera con el salario mínimo legal aplicable para las empresas exportadoras.

1) Destino de la producción: La IME son empresas dedicadas en un $100 \%$ a la exportación para el mercado norteamericano. Las prendas elaboradas en Honduras son exportadas a las grandes cadenas distribuidoras de prendas de vestir de los Estados Uni-

3 Las exportaciones hondureñas de prendas de vestir han gozado de beneficios arancelarios regulados por las siguientes leyes norteamericanas: Caribbean Basin Economic Recovery Act of 1983, Caribbean Basin Economic Recovery Expansion Act of 1990, U.S.-Caribbean Trade Partnership Act of 2000 y Trade Act of 2002.

4 Existen otras textileras como Caracol Knits en el municipio de Potrerillos que abastece a maquiladoras en el municipio de Villanueva; Yangtex y Ssang Bang Wool en el municipio de San Pedro Sula; y Woong Chun en el municipio de Quimistán (Santa Bárbara). dos. Ello no excluye que las prendas después de haber entrado a los EUA regresen a Honduras y cumpliendo los requerimientos de aduanas y el pago de los impuestos correspondientes sean vendidas en el mercado local.

m) Clientes: Los clientes de la IME pueden ser: 1) su casa matriz que sobre la base de un programa de aprovisionamiento global asigna a sus diferentes centros de costos en el Caribe y el resto del mundo pedidos por un determinado período y por una cantidad determinada de prendas. 2) empresas multinacionales de la confección que requieren los servicios de "contratistas" para la elaboración de prendas de vestir en temporadas donde la capacidad propia no cubre la demanda del mercado o para estilos y programas especiales de corta duración. 3) contratistas intermediarios en la cadena entre el productor y las tiendas detallistas de los EUA.

n) Origen de los insumos: La IME importa las telas y los accesorios para la elaboración de las prendas del exterior. Para aprovechar los beneficios arancelarios se importa en gran medida de los EUA o bien de países asiáticos (por ejemplo China y Pakistán). La tela importada puede venir cortada para solo realizar el proceso de unir piezas en Honduras, o bien la tela puede venir entera y se corta aquí sobre la base de los modelos y requerimientos del cliente. Si la tela viene cortada o no, depende exclusivamente del cliente de la IME en EUA quien sobre la base de los beneficios arancelarios que ofrecen diferentes leyes norteamericanas determina cómo hacerlo. ${ }^{3}$ La tela y los accesorios importados son propiedad del cliente. Sin embargo, actualmente existe la tendencia de muchos clientes de exigir que la empresa maquiladora adquiera por sus propios medios todos los insumos (full package). En este caso la empresa en Honduras se aprovisiona por sus propios medios en el mercado internacional y su cliente paga no solo el valor agregado que se le ha dado en Honduras, sino el valor completo.

o) Diversificación: Recientemente se han instalado en estos municipios empresas dedicadas a la elaboración de telas (textileras). Estas empresas como Gildan Activewear (Canadá), ELCATEX (Honduras) y Shing Sung (Corea) se han establecido para explotar los beneficios de las recientes reformas y beneficios adicionales otorgados en el marco de la Caribbean Basin Partnership Act (CBPTA). ${ }^{4} \mathrm{~A}$ través de esta iniciativa se permite la importación a los EUA de algunos productos elaborados con telas producidas en la Cuenca del Caribe pero con hilaza norteamericana. Estas textileras abastecen con telas a otras compañías relacionadas o a otras maquilas no relacionadas.

A partir de las características arriba expuestas se logra identificar que la IME en los municipios de Choloma y Villanueva, en consonancia con la tendencia nacional, son empresas extranjeras (norteamericanas, coreanas en su mayoría) y hondureñas dedicadas a la producción de prendas de vestir para su casa matriz o para otras empresas manufactureras extranjeras. La IME se establece en Honduras, entre otros factores, para aprovechar las ventajas otorgadas por los regímenes especiales arancelarios de los EUA (CBI, CBPTA) y de los regímenes exenciones tributarias de Honduras (ZIP, ZOLI, RIT). Los municipios de Choloma y Villanueva por su cercanía a Puerto Cortés, así como a San Pedro Sula, se desarrollan como centros para la IME. Estos municipios ofrecen grandes cantidades de mano de obra femenina y poco calificada para desarrollar los procesos de corte, costura, planchado y empaque. Las empresas son intensivas en mano de obra poco calificada y emplean en promedio 576 operarios (en el caso 
de Villanueva) y 446 operarios (en el caso de Choloma). No obstante, estos procesos se desarrollan bajo estrictos parámetros de rendimiento y calidad que exigen el detallista y el consumidor norteamericano. Por ser un rubro destinado exclusivamente para la exportación, el empleo y la producción en Honduras depende de los pedidos (de su casa matriz o empresas relacionadas) y/o de los contratos (de su cliente) que resultan de las estrategias de aprovisionamiento global de la industria detallista de los EUA.

\section{LA TRANSFORMACION DE LAS ECONOMIAS LOCALES}

El desarrollo de la IME ha llevado consigo la transformación de Choloma y Villanueva. Estos municipios fueron tradicionalmente economías agrícolas concentradas en la producción de ganado, granos básicos y caña de azúcar (especialmente en Villanueva). Sus economías tradicionales basadas en la tierra estaban dirigidas a abastecer con sus productos agrícolas ciudades como San Pedro Sula y Puerto Cortés. Además servían de paso obligado por carretera o vía férrea en las rutas comerciales entre el Centro y Norte del País, lo que generaba incentivos para la producción de bienes y servicios de la localidad.

Sin embargo, estos municipios experimentaron una transformación sustancial de sus economías locales a partir de los inicios de la década de los años 90 con la aprobación de los programas de ajuste estructural, cuya orientación era la de liberalizar la economía y dinamizar el sector exportador. Estos municipios ubicados muy cerca del principal puerto marítimo de exportación y cruzados por la principal carretera del país que une las ciudades de San Pedro Sula y Tegucigalpa con el mismo puerto, resultaron atractivos para las industrias de ensamble ligero que cobraron auge con la orientación "hacia afuera" de los programas económicos. Bajo este marco de política económica, inversionistas nacionales construyeron parques industriales amparados bajo la Ley ZIP (1987) para albergar las operaciones de las empresas maquiladoras de capital norteamericano, coreano y hondureño entre otras. ${ }^{5}$ Así mismo, el gobierno hondureño realizó la ampliación y modernización de las carreteras que unen a San Pedro Sula y Puerto Cortés con el resto del país.

El primer indicador claro que muestra el impacto social de la IME sobre el municipio es el crecimiento poblacional. Tabla 2 muestra el crecimiento poblacional de las cabeceras municipales de algunos municipios con presencia de la IME. Choloma se destaca por

5 Existía además la Ley y Reglamento de la Zona Libre de Puerto Cortés $(1976,1977)$ que creaba y regulaba las operaciones de esta Zona Libre de capital público y administrada por la Empresa Nacional Portuaria (también de capital público). En esta zona operaron las primeras maquiladoras de la confección de capital norteamericano y asiático. Asi mismo, surgió antes de la Ley ZIP, el Régimen de Importación Temporal en 1984 como un instrumento de exenciones arancelarios para aprovechar los beneficios de la Iniciativa de la Cuenca del Caribe (1983). El RIT aplica para empresas hondureñas dedicadas a la importación de materia prima y bienes de capital para la producción de bienes finales destinados a mercados fuera de la región centroamericana.

6 Instituto Nacional de Estadística: Censo 2001 (XVI Censo de Población y V de de Vivienda, Tegucigalpa, M.D.C., 2002.

7 Para una opinión sobre la contribución de la tasa de natalidad y de la migración poblacional al crecimiento del municipio ver el estudio de FUNDEMUN/AID: Estrategia Participativa para el Desarrollo Integral del Municipio de Choloma. Aquí se argumenta que alrededor del $66 \%$ del crecimiento poblacional de Choloma se atribuye a la natalidad y el resto a la inmigración de otros municipios.

8 Hay que mencionar la situación particular de municipios no maquileros que sirven de "dormitorio" ya que poseen muy pocos puestos de trabajo y su población emigra durante el día a otros municipios. Los municipios del sur del departamento de Cortés (Pimienta, Potrerillos, San Manuel, Santa Cruz, San Antonio y San Francisco) presentan esta situación.

9 Instituto Nacional de Estadística: Vigésima Tercera Encuesta Permanente de Hogares, Tegucigalpa, M.D.C., mayo 2001.

10 Idem.
Tabla 2. Población y tasa de crecimiento: promedio anual de cabeceras municipales

\begin{tabular}{lrrc}
\multicolumn{1}{c}{ Municipio } & $\mathbf{1 9 8 8}$ & $\mathbf{2 0 0 1}$ & $\begin{array}{c}\text { Tasa de creci- } \\
\text { miento anual }\end{array}$ \\
\hline Choloma & 40.554 & 126.402 & 9.1 \\
Villanueva & 12.441 & 32.022 & 7.5 \\
La Ceiba & 71.405 & 126.721 & 4.5 \\
La Lima & 29.805 & 49.48 & 3.9 \\
San Pedro Sula & 298.385 & 483.384 & 3.7 \\
Puerto Cortés & 32.799 & 51.874 & 3.5 \\
Comayagua & 38.656 & 60.078 & 3.4 \\
El Progreso & 62.364 & 94.797 & 3.2 \\
Distrito Central & 648.832 & 819.867 & 1.8
\end{tabular}

Fuente: Instituto Nacional de Estadística: Censo 2001

su crecimiento; su casco urbano experimentó una triplicación de la población en un período de 13 años. Esto representa una tasa promedio de crecimiento del $9 \%$ anual muy por arriba del promedio de todos los centros urbanos del país $(3.1 \%)$ y de todo el departamento de Cortés (4.2\%). ${ }^{6}$ El caso del sector sur del municipio es asintomático de este crecimiento: El Sector López Arellano o Sector Sur del municipio ha crecido de 5 a 32 colonias en diez años, uniéndose prácticamente al casco urbano de San Pedro Sula. Por otro lado, el casco urbano de Villanueva ha seguido muy cerca esta tendencia creciendo a un promedio anual del 7.5\% entre 1988 y 2001.

El crecimiento poblacional de estos municipios ha sido apoyado por la migración de otras regiones del país. ${ }^{7}$ Se trata de migración de habitantes de municipios aledaños y de otros departamentos del país que se movilizan por que han encontrado trabajo en Choloma o Villanueva. Adicionalmente, por la cercanía entre los municipios maquileros existen una proporción importante de operarios que viven, para el caso, en Choloma y trabajan en San Pedro Sula. De esta forma, el crecimiento del municipio se ve también influenciado por el auge de la maquila en otros municipios. El desarrollo de la IME en San Pedro Sula entonces, tiene incidencia en el crecimiento poblacional de otros municipios maquileros. ${ }^{8}$

La existencia de la mayoría de los municipios maquileros en el departamento de Cortés lo convierten, según el Censo 2001 en el departamento del país con mayor migración de hondureños de otros departamentos. ${ }^{9}$ Debido a estas tendencias, hoy en día podemos constatar que Choloma y Villanueva se han convertido en ciudades "medianas" y se ha producido un acercamiento geográfico y económico con San Pedro Sula. Sus poblaciones totales (cabecera y aldeas del municipio) son de 176,789 habitantes para Choloma y 91,613 habitantes para Villanueva. ${ }^{10}$

El crecimiento acelerado de la población, apuntalado por el establecimiento de la IME en su jurisdicción y en otros municipios, ha llevado consigo una serie de cambios en las principales variables sociales. A continuación se destacan algunas áreas sociales de interés y sus respectivos indicadores.

En el campo educativo, una tarea que por los bajos ingresos de la población ha recaído mayormente en el ámbito del Estado, se ha incrementado sustancialmente la población escolar para el nivel preescolar, básico y nivel medio (ver Tabla 3). Esto por un lado, refleja una tendencia positiva que encaja en la tendencia general observada 
Tabla 3. Sistema de Educacion Pública: total de docentes, alumnos y alumnos por docentes en los municipios de Choloma y Villanueva

\begin{tabular}{|c|c|c|c|c|c|c|}
\hline \multirow[b]{2}{*}{ VILLANUEVA } & \multicolumn{3}{|c|}{2001} & \multicolumn{3}{|c|}{2002} \\
\hline & Total docentes & Total alumnos & $\begin{array}{l}\text { Alumnos/ } \\
\text { docentes }\end{array}$ & Total docentes & Total alumnos & $\begin{array}{l}\text { Alumnos/ } \\
\text { docentes }\end{array}$ \\
\hline Total & 263 & 10.57 & 40 & 338 & 19.745 & 58 \\
\hline Nivel Prebásico & 33 & 1.36 & 41 & 45 & 1.42 & 32 \\
\hline Centros de Enseñanza Básica & 36 & 1.609 & 45 & 41 & 1.633 & 40 \\
\hline Nivel Básico & 105 & 4827 & 46 & 252 & 12.096 & 48 \\
\hline Nivel Medio & 89 & 2.774 & 31 & 90 & 4.596 & 51 \\
\hline \multirow[b]{2}{*}{ CHOLOMA } & \multicolumn{3}{|c|}{2001} & \multicolumn{3}{|c|}{2002} \\
\hline & Total docentes & Total alumnos & $\begin{array}{l}\text { Alumnos/ } \\
\text { docentes }\end{array}$ & Total docentes & Total alumnos & $\begin{array}{l}\text { Alumnos/ } \\
\text { docentes }\end{array}$ \\
\hline Total & 581 & 25.57 & 44 & 822 & 36.629 & 45 \\
\hline Nivel Prebásico & 25 & 906 & 36 & 39 & 1.587 & 41 \\
\hline Centros de Enseñanza Básica & 29 & 1.474 & 51 & & & \\
\hline Nivel Básico & 450 & 21.163 & 47 & 671 & 29.388 & 44 \\
\hline Nivel Medio & 77 & 2.027 & 26 & 112 & 5.654 & 50 \\
\hline
\end{tabular}

Fuente: Secretaría de Educación Pública: Estadísticas de la Dirección Departamental de Cortés.

en el departamento de Cortés donde los años de escolaridad han aumentado de 5 años escolares en 1990 a 6 años escolares en 1999. Pero no deja de preocupar por los esfuerzos adicionales en materia de recursos humanos y físicos necesarios generar por parte del gobierno central y las autoridades locales.

Basta con escoger la relación alumno/maestro para apreciar de cerca la problemática. Este indicador ha visto deteriorarse en el corto plazo para los dos municipios. En especial en el nivel medio se observa un aumento significativo de este índice para los dos municipios. En resumen, observamos que la relación alumno/docente ha sobrepasado el nivel de 40 .

Así mismo, otras fuentes muestran que el alfabetismo en los dos municipios se mantiene debajo del promedio departamental (78.1\%): Villanueva con una tasa del 63.2 y Choloma con una tasa del 75.6. ${ }^{11}$

$\mathrm{Al}$ igual que la educación, la situación de la salud sigue registrando tendencias negativas. Para el caso, si se utilizan indicadores de desnutrición infantil, en el año 2001 se registra una tasa de 30.76 y 31.04 para los municipios de Choloma y Villanueva. Esto es preocupante ya que la tasa promedio departamental se ubica en 28.70 y encontramos municipios rurales y no maquileros del departamento de Cortés con tasas de desnutrición menores. ${ }^{12}$

Agregado a esto las investigaciones hechas en el Municipio de Choloma indican una alta incidencia de enfermedades respiratorias y

11 Programa de las Naciones Unidas para el Desarrollo (PNUD): Informe sobre Desarrollo Humano, Honduras 2002. Tegucigalpa, 2002.

12 Programa de las Naciones Unidas para el Desarrollo (PNUD): Informe sobre Desarrollo Humano, Honduras 2002. Tegucigalpa, 2002. Programa de Asignación Familiar (PRAF)/Secretaría de Educación: Octavo Censo de Talla en Niños de Primer Grado Año 2001 (Informe), Tegucigalpa, 2001.

13 FUNDEMUN/AID:Estrategia Participativa para el Desarrollo Integral del Municipio de Choloma, Octubre 2001, pag 37.

14 Idem, pag 37, 38.

15 Las estadísticas del municipio de Choloma indican que solamente un $2.33 \%$ de la población está afectada por un dolencia, contrastando con otros municipios del país cuya afectación llega hasta el $20 \% \wedge$.

16 Municipalidad de Choloma: Censo Socio Económico del Municipio de Choloma. Principales Resultados, 2003. problemas de la piel. Se cuenta con una alta incidencia de tuberculosis e infectados por el virus VIH/SIDA. ${ }^{13}$

Según estas investigaciones el problema de la salud se ha visto agravado por el fenómeno migratorio en el municipio que acelera la transmisión de una serie de enfermedades. ${ }^{14}$ Asimismo, y siguiendo las tendencias en el ámbito nacional la primera y segunda causa de morbilidad son las infecciones respiratorias y gastrointestinales a la que se ve enfrentada en especial la población menor de 5 años. Cabe destacar, sin embargo que las estadísticas de salud del municipio de Choloma y Villanueva no son un reflejo completo de la salud de estos municipios, ya que con la presencia de San Pedro Sula con varios hospitales públicos, muchos habitantes de los municipios maquiladores recurren a éstos en caso de enfermedad. Por consiguiente no se registra la enfermedad en los centros de salud públicos de Choloma y Villanueva. ${ }^{15}$

Respecto a la vivienda, ha sido notorio el crecimiento del casco urbano y la urbanización formal e informal de nuevas tierras orientada a satisfacer las necesidades de vivienda de la población del municipio. Sin embargo, este proceso de urbanización, en muchos casos y de acuerdo a las autoridades municipales, no ha estado acompañado del planeamiento necesario ni de los requerimientos de salubridad y seguridad necesarios. Estas urbanizaciones se han caracterizado por carecer de servicios básicos como agua potable, sistema de alcantarillado sanitario y disposición de desechos sólidos. El último Censo de Población, Vivienda y Establecimientos Comerciales del Área Urbana de Choloma 2002-2003 indica que en sectores de rápido crecimiento como es el Sector Sur de la ciudad, $54.9 \%$ de las viviendas no tienen agua potable, $43.7 \%$ no tiene servicio municipal de recolección de basura y solamente el $54 \%$ de las viviendas están conectadas al alcantarillado sanitario. ${ }^{16}$

Agregado a esto, se ha observado que el crecimiento de las colonias ha estado acompañado por irregularidades que han llevado consigo a problemas judiciales por la propiedad de la tierra. Según las autoridades municipales de Choloma se trata en muchos casos de invasiones a tierras privadas o ejidales que después de urbanizadas originan problemas a los ocupantes de los terrenos y de las viviendas. 
En particular, las autoridades municipales y organizaciones civiles han catalogado de grave la situación surgida para aquella población flotante que no se integra a la ciudad ya que trabaja y duerme durante la semana en el municipio y el fin de semana viaje a sus lugares de origen. Esta población flotante recurre a soluciones habitacionales, "cuarterías", de muy bajo precio y calidad, donde habitan varias personas en un espacio muy limitado. Este segmento de la población trabajadora vive en condiciones de vivienda deplorables.

El Censo de población y vivienda de Honduras del 2001 realizado por el Instituto Nacional de Estadística por su lado indica que la situación de vivienda de los municipios maquiladores no difiere de la situación del departamento de Cortés. Para Choloma las estadísticas indican un promedio de 4.6 personas por vivienda y 4.5 personas por vivienda para Villanueva. Esta situación se muestra ligeramente favorable si comparamos que el promedio nacional se ubica en 5.07 personas por vivienda.

Uno de los impactos sociales que se ha discutido es el empleo generado por la IME. De acuerdo a la Tabla 4 el empleo en la IME ubicada en Choloma y Villanueva llegó a 43,808 empleos y 24,810 empleos respectivamente para el año 1998. Es de importancia, reconocer que no se cuenta con datos sobre el porcentaje de estos operarios que viven en los municipios y por ende el impacto directo sobre la economía local. Sin embargo, el mismo crecimiento poblacional indica que un buen porcentaje de estos operarios vive en los municipios de Choloma y Villanueva demandando una serie de bienes y servicios que ofrece la economía local. Merece la atención indicar que después de una caída del empleo en los años 2001 y 2002, en el año 2003 se observa una leve mejoría en el empleo generado por la IME en ambos municipios.

Ligado al empleo está el impacto de la IME sobre el ingreso. Cabe mencionar que bajo el sistema hondureño de fijación de salarios mínimos por actividad económica indexados con la inflación pasada, el nivel salarial de los operarios de las empresas dedicadas a la producción de bienes y servicios para la exportación (además de los salarios de los empleados de establecimientos financieros y de seguros), se ubica sobre los demás salarios del país. De allí que cabría la suposición que los ingresos reales de los hogares de Choloma y Villanueva ligados a la IME son mayores que los demás hogares no vinculados a la IME. De hecho, si se construye para el período 1998 2002 un promedio para los aumentos del salario mínimo de la IME y para inflación reportada por el $\mathrm{BCH}$, resultaría un aumento salarial promedio mayor que la inflación promedio del período (Tasa de inflación promedio del $10.7 \%$, Tasa de aumento salarial para la IME del $13.1 \%$ ). Esto indicaría, que por lo menos, para todos aquellos

17 En conversación sostenida con la Cámara de Comercio de Choloma se informó sobre la ayuda que está prestando la Cámara de Comercio para financiar las actividades de la Policía Nacional. Se mencionó también la grave crisis de la ganadería cholomeña por el alto grado de inseguridad y de robo de ganado.

18 Existe un grupo representativo de maquilas integradas a grupos empresariales que elaboran la hilaza, la tela y confeccionan las prendas. Lovable (capital hondureño), Gildan Activewear (capital canadiense), Fruit of the Loom (capital norteamericano) son ejemplos de integración de los procesos textiles y de la confección bajo una sola empresa con plantas terrritorialmente dispersas.

19 A través de las entrevistas a los ejecutivos de las maquilas se logró constatar que el único insumo significativo que se adquiere en el país son empaques de plástico y cajas de cartón para el embalaje de las prendas elaboradas. habitantes de Choloma y Villanueva empleados en la IME el ingreso real ha aumentado levemente.

El rápido crecimiento urbano ha significado un deterioro significativo en los niveles de seguridad ciudadana. Los fenómenos de las maras, robos y abigeato se ha acentuado y se manifiesta en especial en las zonas marginales y rurales de los municipios. ${ }^{17}$ A raíz de esto y ante la incapacidad de la Policía Nacional de garantizar un mínimo de seguridad ciudadana las autoridades municipales en Choloma han concertado el cobro de la "tasa de seguridad" para todos sus habitantes. Lo mismo ocurre en Villanueva donde la municipalidad pretende igualmente la introducción de una tasa de seguridad para el financiamiento de medidas policiales necesarias.

Así mismo existen otra serie de factores económicos que se ven influenciados por la actividad maquiladora. Uno de estos factores es la generación de vínculos con la economía local. De acuerdo a la caracterización hecha en el capítulo anterior tanto las empresas maquiladoras que elaboran prendas para clientes (contratistas), así como las maquilas integradas a una casa matriz en los EUA, reciben de su red de proveedores del exterior los insumos necesarios para la manufactura; o bien de las empresas (textileras u otras maquilas) del mismo grupo ubicadas en Honduras y también amparadas en el régimen ZOLI o ZIP. ${ }^{18}$ De allí que cabría esperar que los vínculos que se establezcan con la economía local sean muy pocos.

Actualmente existe la tendencia de clientes norteamericanos de exigir que la empresa maquiladora adquiera por sus propios medios todos los insumos (full package). En este caso la empresa en Honduras se fináncia y aprovisiona por sus propios medios en el mercado internacional y nacional para cumplir con su contrato. No obstante, la información obtenida a través de entrevistas con ejecutivos de la IME indica que son muy pocas las maquiladoras en capacidad de realizar procesos de full package, dado los altos requerimientos de capital de trabajo necesarios y la escasez de insumos para la confección en las industrias locales. ${ }^{19}$ 
Además es necesario considerar los efectos positivos de la IME sobre el comercio, la banca, el transporte y la construcción. Estos servicios han resultado rubros ganadores del auge de la IME y en especial del gasto en consumo e inversión que realizan los operarios de la maquila y demás habitantes involucrados indirectamente con la IME.

Cabría entonces solamente esperar que los impulsos que envía la IME a la economía local se circunscriban al consumo e inversión que surge de los salarios y remuneraciones de los operarios. De allí surgen demandas por servicios bancarios, de comercio de transporte, de construcción de viviendas así como de algunos servicios que las maquilas y los administradores de los parques industriales requieren para el mantenimiento y reparación de las naves industriales y maquinaria. Indudablemente, que dada la magnitud de la IME, medida sobre la base de la generación de empleo, incremento de la migración laboral, utilización de recursos y bienes públicos así como sobre la base de la inversión privada en infraestructura, existen muchas potencialidades que no son explotadas, dada las características del régimen local bajo el cual se amparan y dada las condiciones internacionales de integración con las casas matrices. Los impulsos generados por la IME para el desarrollo local podrían ser mayores, en la medida en que la IME implemente los procesos de full-package y la industria local pueda ofrecer insumos en las cantidades requeridas por las maquilas y a precios internacionalmente competitivos. ${ }^{20}$

La IME trabaja bajo altos requerimientos de calidad. La producción está minuciosamente regulada por los requerimientos de tiempo por tipo de operación. En este sentido, los operarios son entrenados para que en un período relativamente corto eleven la intensidad del trabajo al nivel deseado. Además, se ejerce en la producción un estricto control de calidad a través de inspecciones concurrentes y a posteriori al producto en proceso y al producto terminado. Sin duda alguna, este es un punto de relevancia ya que eleva los niveles de productividad de la mano de obra a las mejores prácticas de la industria del vestuario en el ámbito mundial.

Sin embargo, las actividades transferidas a la IME en Honduras son actividades intensivas en mano de obra y requieren de muy poca formación profesional. Los operarios son en su mayoría personal femenino con poca escolaridad y desarrollan actividades básicamente de costura, planchado, inspección y empacado. De acuerdo a las investigaciones hechas son muy pocas las maquilas que se desvían de estas operaciones tradicionales generando por lo tanto muy poco valor agregado. Dadas estas características se puede afirmar que los procesos de transferencia y cambio tecnológico al recurso humano y a otros rubros económicos son limitados. ${ }^{21}$

El Programa de la Naciones Unidas para el Desarrollo (PNUD) ha venido elaborando un Índice de Desarrollo Humano (IDH) para Honduras que arroja luz sobre la situación nacional y sobre el desarrollo de los departamentos y municipios. ${ }^{22}$ En este sentido, el PNUD reporta que "el análisis por departamento pone en evidencia el patrón

20 Uno de los efectos evidentes de la IME hacia la economías locales son el surgimiento de una cantidad considerable de "negocios ambulantes"que ofrecen alimentos, ropa y enseres para el hogar cerca de los parques industriales. La municipalidad de Villanueva ha intentado regular estas ventas ya que no tributan y además obstaculizan las vías de comunicación. Se estima que para el caso en Villanueva 500 vendedores ambulantes ofrecen sus productos diariamente.

21 Para ver resultados a nivel centroamericano y mexicano referidos a la transferencia de tecnología y desarrollo tecnológico local ver Buitelaar, Rudolf M./Padilla, Ramón/ Urrutia, Ruth (1999): Industria Maquiladora y cambio técnico, en Revista de la CEPAL, abril 1999.

22 Programa de las Naciones Unidas para el Desarrollo (PNUD): Informe sobre Desarrollo Humano, Honduras: 1999, Tegucigalpa, 1999.

23 Programa de las Naciones Unidas para el Desarrollo (PNUD): Informe sobre Desarrollo Humano, Honduras: 2002. Tegucigalpa, 2002. diferenciador del desarrollo nacional, en el que los departamentos costeros, sobre todo del norte del país y aquellos donde se ubican los centros urbanos más grandes, presentan los mayores logros”. 23 Es decir, que los municipios maquileros se ubican en el segmento correspondiente a desarrollo humano medio, medido éste a través de la dimensión educativa, productiva y de salud.

A través de los indicadores económicos y sociales descritos anteriormente se puede concluir en lo siguiente: El empleo y el ingreso generado directamente por la IME son factores de gran relevancia para el desarrollo económico local. Estos ingresos han generado el sustento de miles de operarios y ha incorporado al mercado laboral a una cantidad significativa de mano de obra femenina. El nivel salarial de la IME se ubica por disposición legal arriba de los salarios de otros rubros de producción y los cálculos indican que para el período 1998 2002 el incremento promedio del salario mínimo de la IME se ubicó arriba del incremento de la tasa de inflación para el mismo período. Dada los altos requerimientos de rendimiento y calidad en la producción, la mano de obra empleada trabaja bajo estándares mundiales de manufactura, elevando la competitividad de la mano de obra. Se ha logrado además dinamizar muchos sectores económicos tradicionalmente ausentes o con muy poco desarrollo como ser la banca, el comercio, transporte y la construcción.

No obstante el nivel alcanzado, las tendencias que se mostraron anteriormente ponen en duda la sustentabilidad del desarrollo local de estos municipios. A pesar de que estos municipios poseen un desarrollo humano medio según el PNUD, la educación, salud, seguridad ciudadana y vivienda de los municipios maquiladores muestran características preocupantes. El crecimiento de la población escolar, el aumento de las enfermedades y dolencias, el incontrolable crecimiento urbano sin los niveles mínimos de agua y saneamiento, así como el incremento de la inseguridad ciudadana son algunos indicadores que muestran el impacto social negativo.

Por otro lado, la economía local ha logrado integrarse limitadamente al desarrollo de la IME. Dadas las relaciones de las maquilas con redes mundiales de aprovisionamiento y su integración con las casas matrices en los EUA se establecen muy pocos eslabonamientos con la economía local y nacional. Adicionalmente, las operaciones realizadas en la IME por los operarios requieren de mano de obra poco calificada que realiza en su mayoría actividades de costura, planchado, inspección y empacado, generadoras de poco valor agregado.

\section{BIBLIOGRAFIA}

1. Asociación Hondureña de Maquiladores (AHM): Directorios 1996-2003.

2. Banco Interamericano de Desarrollo (BID): Programa de Inversiones en Agua Potable y Saneamiento (H0-0072).

3. Buitelaar, Rodolfo M/Padilla, Ramón/Urrutia, Ruth: Industria Maquiladora y cambio técnico, en: revista de la CEPAL, abril 1999.

4. Congreso Nacional de la República: Código del Trabajo (Decreto No. 189).

5. Congreso Nacional de la República: Ley Marco del Sector Agua Potable y Saneamiento (Dictamen), septiembre 2002. 134-90).

6. Congreso Nacional de la República: Ley de Municipalidades (Decreto No.

7. FIDE: Agenda de Competitividad de Honduras: La Industria Textil y de Confección en Honduras-Condiciones Competitivas del Valle de Sula, Tegucigalpa, marzo 2003.

8. FUNDEMUN/AID: Estrategia participativa para el desarrollo integral del municipio de Choloma, Octubre 2001.

9. Inter-American Development Bank (IADB): Sub National Finance and Development (A Draft Operational Strategy for Region II, Mexico, Central America, Belize, Panama, Dominican Republic, Haiti), 1998.

10. Instituto Nacional de Estadística (INE): Vigésimo Tercera Encuesta Permanente de Hogares, 2001.

11. Instituto Nacional de Estadística (INE): Censo 2001 (Tomo 1). 
12. Llisterri, Juan José: Competitividad y Desarrollo Económico Local Nuevas Oportunidades Operativas (Documento de Discusión)

13. Municipalidad de Choloma: Censo Socio Económico del Municipio de Choloma, Principales Resultados, Choloma 2003.

14. Municipalidad de Villanueva: Plan de Arbitrios 2003.

15. Municipalidad de Villanueva: Perfil del Municipio de Villanueva.

16. Municipalidad de Villanueva: Memoria Municipal 1996.

17. Programa de Asignación Familiar/Secretaría de Educación: Octavo Censo de Talla en Niños de Primer Grado Año 2001, Tegucigalpa 2001.

18. Programa de la Naciones Unidas para el Desarrollo (PNUD): Informe sobre Desarrollo Humano 1999.

19. Programa de la Naciones Unidas para el Desarrollo (PNUD): Informe sobre Desarrollo Humano 2002.

20. Secretaría de Educación Pública: Estadísticas de la Dirección Departamental de Cortés.
21. United Nations Conference on Trade and Development (UNCTAD): World Investment Report 2001-Promoting Linkages, New York 2001.

22. Worldwide Responsible Apparel Production (WRAP): Manual de Autoevalución e Inspección para las Instalaciones de Producción.

ENTREVISTAS REALIZADAS

Gerente Administrativo Municipalidad de Choloma

Gerente Administrativo Municipalidad de Villanueva

Gerente Agua y Saneamiento de Villanueva

Gerente Dirección Municipal de Aguas de Choloma

Gerente de Planeamiento Urbano Municipalidad de Choloma

Gerente de Planeamiento Urbano Municipalidad de Villanueva

Gerente de Acción Comunitaria Municipalidad de Villanueva

Gerente de Promoción Comunitaria Municipalidad de Villanueva

Gerente de la Cámara de Comercio e Industrias de Choloma

Gerente de la Cámara de Comercio e Industrias de Villanueva 